\title{
Commentary
}

\section{Sedentary lifestyle among office workers and coronary heart disease risk factors due to the COVID-19 quarantine}

\author{
Mahboubeh Ghayour Najafabadi ${ }^{\mathrm{a}}$, Amir Sobhrakhshan Khah ${ }^{\mathrm{b}, *}$ and Mitch Rostad \\ ${ }^{a}$ Department of Motor Behavior, Faculty of Physical Education and Sport Science, University of Tehran, Tehran, \\ Iran \\ ${ }^{\mathrm{b}}$ Sepehr Heart center Baharloo hospital, Tehran University of Medical Sciences, Tehran, Iran \\ c Organ Preservation Alliance, Berkeley, CA, USA
}

Received 26 August 2020

Accepted 5 September 2020

\begin{abstract}
.
The COVID-19 pandemic has affected the entire world. It is difficult to follow protocols and regulations set forth by governments, designed by the World Health Organization. The most common protocol set forth by governments is quarantining at home. Many occupations must stay home to comply with this protocol. Among these occupations, office workers are the most common group to comply and work from home. This has led to a lack of daily movement and increased sedentary lifestyle, which has made employees prone to developing coronary heart disease (CHD). Additionally, obesity is a known risk factor for this group. This commentary presents feasible protocols aimed at helping home-based office workers stay healthy and decrease the risk of developing CHD.
\end{abstract}

Keywords: Coronavirus, pandemic, health, occupation, home-based

\section{Introduction}

A sedentary life style among office workers has increased from 26 to $38 \mathrm{~h}$ per week between 1965 and 2009 in the USA expressing alarming prospects for 2030 [1]. Many studies have shown a correlation between sedentary behavior and increased risks for cardiovascular disorders, type II diabetes, obesity, cancer (colon and breast), high blood pressure, musculoskeletal complaints and psychological disorders

\footnotetext{
*Address for correspondence: Amir Sobhrhakhshan Khah, Sepehr Heart center Baharloo hospital, Tehran University of Medical Sciences, Tehran, Iran. E-mail: amirrakhshankhah@gmail. com.
}

(depression and anxiety) [2, 3]. Sedentary behavior both during work and leisure time is estimated to be responsible for about one-third of annual deaths due to coronary heart disease (CHD) in the USA (each year 1.9 million people die a premature death because of a sedentary lifestyle - based on WHO statistics). There is no standard definition of sedentary lifestyle and sedentary behavior, however, sedentary behavior refers to any waking activity characterized by a low energy expenditure while sitting or reclining. What is even worse is that people with $\mathrm{CHD}$, for whom a sedentary lifestyle may have been a contributing factor to their disease, receive medical advice based on academic recommendations that often discourages 
them to perform physical activity out of fear that it may exacerbate their heart condition [4].

The current COVID-19 pandemic is especially concerning for office workers that must stay at home. They more easily engage in a sedentary lifestyle because their lives are devoid of the most basic activities of daily living including dressing in professional attire and transporting themselves to work [5]. Studies show that as a person's life becomes more sedentary, their social, cognitive, and physical health will deteriorate and they will be at an increased risk of comorbidities like CHD [6, 7]. These problems are dramatic and are seen among office workers that telework during the COVID-19 quarantine. To ameliorate the aforementioned problem and consequence, we recommend several protocols [8].

\section{Physical protocols: $150-300$ min per week aerobic exercise and two times per week strength exercise}

Aerobic exercise: march in place, climbing up and down steps, dance, online aerobic exercise

Strength exercise: squat to chair, pushups on the wall or the floor, isometric exercise with body weight, online exercise.

Physical activity: break up sitting, walk while speaking on the phone (especially for work), stand while eating, cook food, wash and dry dishes, play video interactive movement games found on many electronic home gaming consoles, neck side stretch [9].

Benefit: physical activity has a significant positive influence on comorbidities like CHD, by strengthening the heart and bones, lower blood pressure and cholesterol, decrease pain symptoms, weight loss, and improve feeling of wellness [10].

\section{Mental protocols}

Cognitive stimulation (using apps or stimulating mental exercises, especially for people with previous cognitive impairment, e.g. sudoku): meditation, reading, writing, listening to calm music, drawing [11].

Benefit: Mental exercise helped patients with symptoms of CHD develop a deeper cognitive awareness to identify negative stress-related thought patterns and instead, redirect their attention to the positivity of the present moment [12].

\section{Social protocols}

To prevent social isolation: stay connected with relatives and friends using technology, maintain you spiritual and religious practice, watch positive videos and movies.

Benefit: Social activity has been tied to biological mechanisms including the effects of psychologic stimuli on the autonomic nervous system that can decrease stress and depression [13].

\section{Conclusion}

COVID-19 quarantined office workers must be aware that their lack of typical daily activity can cause significant physical health deterioration. The simple protocols that we recommend in this work can easily and enjoyably be integrated into a person's life and improve physical health while alleviating consequences typically seen in a sedentary lifestyle.

\section{Acknowledgments}

This study is supported by University of Tehran (Grant number: 30701.1.4).

\section{Conflict of interest}

The authors declared no potential conflicts of interest with respect to the research, authorship, and/or publication of this article.

\section{References}

[1] León-Latre M, Moreno-Franco B, Andrés-Esteban EM, Ledesma M, et al. Sedentary lifestyle and its relation to cardiovascular risk factors, insulin resistance and inflammatory profile. Rev Esp Cardiol (English Edition). 2014;67(6): 449-55.

[2] Mainous AG, Tanner RJ, Rahmanian KP, Jo A, et al. Effect of sedentary lifestyle on cardiovascular disease risk among healthy adults with body mass indexes 18.5 to $29.9 \mathrm{~kg} / \mathrm{m} 2$. Am J Cardiol. 2019;123(5):764-8.

[3] Shariat A, Cardoso JR, Cleland JA, Danaee M, et al. Prevalence rate of neck, shoulder and lower back pain in association with age, body mass index and gender among Malaysian office workers. Work. 2018;(Preprint):1-9.

[4] Cheng L, Pohlabeln H, Ahrens W, Lauria F, et al. Crosssectional and longitudinal associations between physical activity, sedentary behaviour and bone stiffness index across weight status in European children and adolescents. Int J Behav Nutr Phys Act. 2020;17:1-13. 
[5] Shariat A, Hakakzadeh A, Cleland J. Home-based exercise note in Covid-19 quarantine situation for office workers: A commentary. Work. 2020;(Preprint):1-2.

[6] Parvin N, Nath MC. Comparison of Cardiovascular Risk Factors between Sedentary and Non Sedentary Workersin Rajshahi Metropolitan Area. J Dent Med Scie. 2020;19(2):41-46.

[7] Vaccarino V, Badimon L, Bremner JD, Cenko E, et al. Depression and coronary heart disease: 2018 position paper of the ESC working group on coronary pathophysiology and microcirculation. Eur Heart J. 2020;41(17):1687-96.

[8] Soheili S, Shariat A, Anastasio A. Modification of existing occupational therapeutic protocols in response to new normal life after COVID 19: Letter to the Editor. Work. 2020;(Preprint):1-2.

[9] Hammami A, Harrabi B, Mohr M, Krustrup P. Physical activity and coronavirus disease 2019 (COVID-19): specific recommendations for home-based physical training. Manag Sport Leis. 2020;1-6.

[10] Amatriain-Fernández S, Murillo-Rodríguez ES, Gronwald T, Machado S, et al. Benefits of physical activity and physical exercise in the time of pandemic. Psychol Trauma. 2020;12(S1):S264.

[11] Golberstein E, Wen H, Miller BF. Coronavirus disease 2019 (COVID-19) and mental health for children and adolescents. JAMA Pediatr. 2020;

[12] Parswani MJ, Sharma MP, Iyengar SS. Mindfulness-based stress reduction program in coronary heart disease: A randomized control trial. Int J Yoga. 2013;6(2):111.

[13] Greenwood DC, Muir KR, Packham CJ, Madeley RJ. Coronary heart disease: a review of the role of psychosocial stress and social support. J Public Health Med. 1996;18(2):221-31. 